PROCEEDINGS OF THE

AMERICAN MATHEMATICAL SOCIETY

Volume 133, Number 5, Pages 1283-1290

S 0002-9939(04)07825-6

Article electronically published on December 15, 2004

\title{
MOMENTS OF THE PROBABILITY DENSITY FUNCTIONS OF ERROR TERMS IN DIVISOR PROBLEMS
}

\author{
YUK-KAM LAU AND KAI-MAN TSANG
}

(Communicated by David E. Rohrlich)

\begin{abstract}
We derive an explicit formula for the moments of the probability density function of a class of functions. An application of this shows that the density function of the error term in the Pilz divisor problem is asymmetric.
\end{abstract}

\section{INTRODUCTION}

The error term $\Delta(x)$ in the Dirichlet divisor problem has been studied extensively by numerous authors. In the paper [3], Heath-Brown pioneered the study of the limit distribution of $\Delta(x)$. Among other things, he showed that $\Delta(x)$ has a limiting distribution and the probability density function decays rapidly. Heath-Brown's method actually applies to a rather general type of functions $F(t)$, which satisfy the following

Hypothesis (H). There exists a sequence $a_{1}(t), a_{2}(t), \cdots$ of continuous real-valued functions of period 1 such that

$$
\lim _{N \rightarrow \infty} \limsup _{T \rightarrow \infty} \frac{1}{T} \int_{0}^{T} \min \left\{1,\left|F(t)-\sum_{n \leq N} a_{n}\left(\gamma_{n} t\right)\right|\right\} d t=0
$$

where $\gamma_{1}, \gamma_{2}, \cdots$ are nonzero constants which are linearly independent over $\mathbb{Q}$.

Remark. The condition of taking $\min \{1,|\cdot|\}$ in $(\mathrm{H})$ is the weakest among all measurements $\min \left\{1,|\cdot|^{\beta}\right\}(\beta>0)$, in view of Hölder's inequality (or see [4. p. 362]).

One of the main results in $[3]$ is the following.

Theorem A ([3, Theorem 5]). If $F(t)$ satisfies Hypothesis $(\mathrm{H})$ with the $a_{n}(t)$ 's satisfying the conditions:

(C.1) $\int_{0}^{1} a_{n}(t) d t=0 \quad(n \in \mathbb{N})$,

(C.2) $\sum_{n=1}^{\infty} \int_{0}^{1} a_{n}(t)^{2} d t<\infty$,

(C.3) $\max _{t \in[0,1]}\left|a_{n}(t)\right| \ll n^{1-\mu}$ and $\lim _{n \rightarrow \infty} n^{\mu} \int_{0}^{1} a_{n}(t)^{2} d t=\infty$

where $\mu>1$ is a constant, then

$$
T^{-1} \text { meas }\{t \in[0, T]: F(t) \in I\} \rightarrow \int_{I} f(\alpha) d \alpha
$$

Received by the editors November 11, 2003.

2000 Mathematics Subject Classification. Primary 11N60.

(C)2004 American Mathematical Society Reverts to public domain 28 years from publication 
as $T \rightarrow \infty$. Furthermore, the density function $f(\alpha)$ satisfies

$$
\frac{d^{k}}{d \alpha^{k}} f(\alpha) \ll_{A, k}(1+|\alpha|)^{-A}
$$

for $k=0,1,2, \cdots$ and any constant $A$ and $f(\alpha)$ extends to an entire function on $\mathbb{C}$.

From the high decaying rate of $f(\alpha)$, it is apparent that all the power moments of $f(\alpha)$ exist. But this does not imply that the power moments of $F(t)$ converge. Heath-Brown [3, Theorem 6] established that if $\lim _{T \rightarrow \infty} T^{-1} \int_{0}^{T}|F(t)|^{K} d t$ is finite, then for any positive integer $k<K, \lim _{T \rightarrow \infty} T^{-1} \int_{0}^{T} F(t)^{k} d t$ exists. In such cases, it is known that

$$
\lim _{T \rightarrow \infty} T^{-1} \int_{0}^{T} F(t)^{k} d t=\int_{-\infty}^{\infty} \alpha^{k} f(\alpha) d \alpha
$$

The variance (second power moment) in [2] was also computed in this way.

In this note, we obtain a quite explicit formula for all the integral power moments of the density function, without the knowledge of the power moments of $F(t)$. As a consequence, we can say something about the symmetry of the density functions of some well-known error terms in number theory, including $\Delta(x)$ and $\Delta_{3}(x)$, the error terms in the Dirichlet and Pilz divisor problems respectively. (See Corollary 4.)

Theorem 1. Under the assumptions in Theorem $\AA$, we have, for any $k \in \mathbb{N}$,

$$
\int_{-\infty}^{\infty} \alpha^{k} f(\alpha) d \alpha=\sum_{1 \leq r \leq k} \sum_{\substack{l_{1}, \ldots, l_{r} \geq 1 \\ l_{1}+\cdots+l_{r}=k}} \frac{k !}{l_{1} ! l_{2} ! \cdots l_{r} !} \sum_{1 \leq n_{1}<\cdots<n_{r}} \prod_{i=1}^{r} A\left(n_{i}, l_{i}\right),
$$

where

$$
A(n, l)=\int_{0}^{1} a_{n}(t)^{l} d t
$$

The infinite series in (1.1) converges absolutely.

Remarks. (i) As $A(n, 1)=0$ for all $n$ by (C.1), the first moment of $f(\alpha)$ always vanishes, that is,

$$
\int_{-\infty}^{\infty} \alpha f(\alpha) d \alpha=0
$$

(ii) The function $f(\alpha)$ is symmetric if and only if

$$
\int_{-\infty}^{\infty} \alpha^{k} f(\alpha) d \alpha=0
$$

for all positive odd integers $k$. Hence it is known that the density function of $x^{-1 / 4} \Delta(x)$ is asymmetric, since the third power moment $X^{-1} \int_{0}^{X}\left(x^{-1 / 2} \Delta\left(x^{2}\right)\right)^{3} d x$ is nonzero (see [5]). For $\Delta_{3}(x)$, however, we cannot draw a similar conclusion in this way since none of the odd power moments higher than the first is known for $\Delta_{3}(x)$. Using our Theorem 1 , we can show that

$$
\int_{-\infty}^{\infty} \alpha^{3} f(\alpha) d \alpha \neq 0
$$


for the density function $f(\alpha)$ of $x^{-1 / 3} \Delta_{3}(x)$. Thus $x^{-1 / 3} \Delta_{3}(x)$ also has an asymmetric density function (see Corollary 4 ). More generally, concerning the symmetry of the density function $f(\alpha)$ of a certain $F(t)$, we have the following theorems.

Theorem 2. If each $a_{n}(t)$ in Theorem $\$$ is of the form $a_{n}(t)=b(n) \cos \left(2 \pi t+\phi_{n}\right)$ where $b(n), \phi_{n} \in \mathbb{R}$, then all the odd power moments of $f(\alpha)$ are equal to zero and hence $f(\alpha)$ is symmetric.

Theorem 3. Suppose for each $n \in \mathbb{N}, a_{n}(t)$ is of the form

$$
a_{n}(t)=\sum_{m=1}^{\infty} b_{n}(m) \cos (2 \pi m t-\theta)
$$

where $\theta \in[-\pi, \pi]$ and the series is absolutely convergent. If the assumptions in Theorem $\triangle$ hold, then

(a) when $\theta= \pm \pi / 2$, the density function $f(\alpha)$ is symmetric;

(b) when $\theta \neq \pm \pi / 2$ and when all $b_{n}(m)$ are nonnegative and satisfy $b_{n}\left(m_{1}\right) b_{n}\left(m_{2}\right) b_{n}\left(m_{1}+m_{2}\right) \neq 0$ for some $n, m_{1}, m_{2} \in \mathbb{N}$, then the density function $f(\alpha)$ is asymmetric.

Theorem 2 is straightforward, since by (1.2),$A(n, l)=0$ for any positive odd integer $l$, and for $l_{1}+l_{2}+\cdots+l_{r}$ equal to an odd integer, at least one $l_{i}$ must be odd.

To deduce Theorem 3, we first evaluate each of $A(n, l)$. Let $E_{l}$ be the set of all maps $\sigma$ sending $\left(x_{1}, \cdots, x_{l}\right)$ in $\mathbb{R}^{l}$ to one of $x_{1} \pm \cdots \pm x_{l}$, and let $n_{-}(\sigma)$ denote the number of minus signs in $\sigma\left(x_{1}, \cdots, x_{l}\right)$. Using the identity $\prod_{i=1}^{l} \cos A_{i}=$ $2^{1-l} \sum_{\sigma \in E_{l}} \cos \left(\sigma\left(A_{1}, \cdots, A_{l}\right)\right)$, we see that

$$
A(n, l)=2^{1-l} \sum_{\sigma \in E_{l}} \sum_{\substack{m_{1}, \cdots, m_{l} \geq 1 \\ \sigma\left(m_{1}, \cdots, m_{l}\right)=0}} \prod_{i=1}^{l} b_{n}\left(m_{i}\right) \cdot \cos \left(\left(l-2 n_{-}(\sigma)\right) \theta\right) .
$$

For $\theta= \pm \pi / 2$, it is clear that $A(n, l)=0$ for all odd $l$. Hence for all positive odd $k$,

$$
\int_{-\infty}^{\infty} \alpha^{k} f(\alpha) d \alpha=0,
$$

since for $l_{1}+\cdots+l_{r}=k$, at least one $l_{i}$ is odd. Thus $f(\alpha)$ is symmetric. On the other hand, under the conditions in (b), the third power moment

$$
\int_{-\infty}^{\infty} \alpha^{3} f(\alpha) d \alpha=\frac{\cos \theta}{4} \sum_{n=1}^{\infty} \sum_{m_{1} \pm m_{2} \pm m_{3}=0} \prod_{i=1}^{3} b_{n}\left(m_{i}\right) \neq 0 .
$$

Hence, the density function $f(\alpha)$ is asymmetric.

Remark. In view of the proof, the conditions imposed on the coefficients $b_{n}(m)$ in Theorem 3 (b) can be relaxed to the nonvanishing of the multiple sum in (1.3).

Corollary 4. Let $\Delta(x)$ and $\Delta_{3}(x)$ be the error terms in the Dirichlet divisor problem and the Pilz divisor problem respectively. Then both of their density functions are asymmetric.

The corollary follows from the fact that the Voronoi series approximations for $x^{-1 / 4} \Delta(x)$ and $x^{-1 / 3} \Delta_{3}(x)$, as can be seen in [3], for example, are of the type in Theorem 3(b). 
In [3], it is also shown that the odd power moments

$$
\int_{0}^{X} \Delta(x)^{k} d x \sim \beta_{k} X^{1+k / 4}
$$

for $1 \leq k \leq 9$, with undetermined constants $\beta_{k}$. Recently Zhai [6] reported that these constants are all positive (except $\beta_{1}=0$ ), and he conjectured that this is also the case for all odd $k \geq 11$. We now, however, give the following two examples to illustrate the possible peculiar features of the density function.

Example 1. Let $K$ be any positive integer and let

$$
a_{n}(t)=\mu(n)^{2} n^{-3 / 4}(\cos (2 \pi t)+\cos (4 \pi K t)) .
$$

Define $F(t)=\sum_{n=1}^{\infty} a_{n}(\sqrt{n} t)$. We shall see that the $k$ th power moment of $f$ is equal to zero for $k=1,3, \cdots, 2 K-1$ but is positive for $k=2 K+1$.

Let $1 \leq l \leq 2 K+1$ be odd and $m_{i} \in\{1,2 K\}$. Each $\sigma\left(m_{1}, \cdots, m_{l}\right)\left(\sigma \in E_{l}\right)$ can be written as

$$
\sigma\left(m_{1}, \cdots, m_{l}\right)= \pm 2 K \sigma_{1}(1, \cdots, 1) \pm \sigma_{2}(1, \cdots, 1)
$$

for certain $\sigma_{1} \in E_{r}$ and $\sigma_{2} \in E_{s}$ with $r+s=l$. Since $\left|\sigma_{2}(1, \cdots, 1)\right| \leq s \leq l$, $\sigma\left(m_{1}, \cdots, m_{l}\right)=0$ only if $l=2 K+1, r=1$ and $\sigma_{2}(1, \cdots, 1)=2 K$. Hence $\int_{0}^{1} a_{n}(t)^{l} d t$ is equal to zero for all odd $l \leq 2 K-1$ and is positive for $l=2 K+1$.

Example 2. We construct below a function $F(t)=\sum_{n=1}^{\infty} a_{n}(\sqrt{n} t)$ whose third power moment is positive while its fifth power moment is negative.

Let

$$
a_{n}(t)=\mu(n)^{2} n^{-3 / 4}(\cos (2 \pi t-\pi / 4)+b \cos (4 \pi t-\pi / 4)+\cos (16 \pi t-\pi / 4))
$$

where $b>0$ is a large number to be determined later. Direct computation shows that $A(n, 3)=\frac{3}{4} b \mu^{2}(n) n^{-9 / 4} \cos (\pi / 4)$, and the third power moment equals

$$
\frac{3 b}{4 \sqrt{2}} \frac{\zeta(9 / 4)}{\zeta(9 / 2)}>0
$$

To evaluate the fifth power moment, we note that $A(n, 2)=\left(1+\frac{1}{2} b^{2}\right) \mu^{2}(n) n^{-3 / 2}$. From Theorem 1 the fifth power moment is thus equal to

$$
\sum_{n \neq n^{\prime}} \frac{5 !}{3 ! 2 !} A(n, 2) A\left(n^{\prime}, 3\right)+\sum_{n=1}^{\infty} A(n, 5) .
$$

The first double sum is $O\left(b^{3}\right)$. Up to permutations, for each $\sigma \in E_{5}, \sigma\left(m_{1}, \cdots, m_{5}\right)$ is of the form

$\pm\left(m_{1}+m_{2}+m_{3}+m_{4}-m_{5}\right) \quad$ or $\quad \pm\left(m_{1}+m_{2}+m_{3}-m_{4}-m_{5}\right) \quad$ or $\quad m_{1}+\cdots+m_{5}$.

When $m_{i} \in\{1,2,8\}$, the first form equals 0 only for the case $m_{1}=\cdots=m_{4}=2$ and $m_{5}=8$; the second form takes zero with at most three $m_{i}$ 's equal to 2 , while the last form never vanishes. This yields

$$
A(n, 5)=\left(\frac{5 b^{4}}{16} \cos \left(\frac{3 \pi}{4}\right)+O\left(b^{3}\right)\right) \frac{\mu^{2}(n)}{n^{15 / 4}} .
$$

Therefore, when $b$ is large enough, the fifth power moment of the corresponding density function is equal to

$$
-\frac{5}{16 \sqrt{2}} \frac{\zeta(15 / 4)}{\zeta(15 / 2)} b^{4}+O\left(b^{3}\right)<0 .
$$




\section{Some PREPARATIONS}

We first prove a lemma for later use, and meanwhile, show that the infinite sum in (1.1) is absolutely convergent.

Lemma 1. For any integer $k \geq 1$, the multiple sum in (1.1) converges absolutely. Let $c_{k}$ be the value of this multiple sum. Then,

$$
\lim _{N \rightarrow \infty} \lim _{T \rightarrow \infty} T^{-1} \int_{0}^{T}\left(\sum_{n \leq N} a_{n}\left(\gamma_{n} t\right)\right)^{k} d t=c_{k} .
$$

To prove Lemma 1, we approximate each $a_{n}(t)$ by the trigonometric polynomial $\sigma_{n, M}(t)$, which is the convolution of $a_{n}$ with the Fejér kernel, that is,

$$
\sigma_{n, M}(t)=\int_{0}^{1} a_{n}(t+v) \frac{1}{M}\left(\frac{\sin \pi M v}{\sin \pi v}\right)^{2} d v=\sum_{|m| \leq M} c_{n, m} e(m t),
$$

where $c_{n, m}=(1-|m| / M) \hat{a}_{n}(m)$ and $e(y)=e^{2 \pi i y}$. It is well known that

$$
\left\|a_{n}-\sigma_{n, M}\right\| \rightarrow 0 \text { as } M \rightarrow \infty .
$$

Here $\|\cdot\|$ denotes the supremum norm on $[0,1]$. Moreover, from (2.1) and (C.3), we see that

$$
\left\|\sigma_{n, M}\right\| \leq\left\|a_{n}\right\| \ll 1 \quad \text { for } \quad n \in \mathbb{N} .
$$

Using $a^{k}-b^{k} \ll_{k}|a-b|\left(|a|^{k-1}+|b|^{k-1}\right)$ and (2.3), we obtain

$$
\begin{array}{ll} 
& \left(\sum_{n \leq N} a_{n}\left(\gamma_{n} t\right)\right)^{k}-\left(\sum_{n \leq N} \sigma_{n, M}\left(\gamma_{n} t\right)\right)^{k} \\
\ll_{k} & \left(\sum_{n \leq N}\left\|a_{n}\right\|\right)^{k-1} \sum_{n \leq N}\left|a_{n}\left(\gamma_{n} t\right)-\sigma_{n, M}\left(\gamma_{n} t\right)\right| \\
\ll_{k} \quad & N^{k-1} \sum_{n \leq N}\left\|a_{n}-\sigma_{n, M}\right\| .
\end{array}
$$

Let us write $m_{T}(f)=T^{-1} \int_{0}^{T} f(t) d t$ for short. Then

$$
\begin{aligned}
& m_{T}\left(\left(\sum_{n \leq N} a_{n}\left(\gamma_{n} t\right)\right)^{k}\right)-m_{T}\left(\left(\sum_{n \leq N} \sigma_{n, M}\left(\gamma_{n} t\right)\right)^{k}\right) \\
\ll_{k} \quad & N^{k-1} \sum_{n \leq N}\left\|a_{n}-\sigma_{n, M}\right\| .
\end{aligned}
$$

Let

$$
S_{M}(n, l)=\int_{0}^{1} \sigma_{n, M}(t)^{l} d t,
$$

and define

$$
S_{M}^{(k)}(N)=\sum_{1 \leq r \leq k} \sum_{\substack{l_{1}, \ldots, l_{r} \geq 1 \\ l_{1}+\cdots+l_{r}=k}} \frac{k !}{l_{1} ! \cdots l_{r} !} \sum_{n_{1}<\cdots<n_{r} \leq N} \prod_{i=1}^{r} S_{M}\left(n_{i}, l_{i}\right) .
$$


Then we have

Lemma 2. Given any positive integers $k, N$ and $M$,

$$
m_{T}\left(\left(\sum_{n \leq N} \sigma_{n, M}\left(\gamma_{n} t\right)\right)^{k}\right) \rightarrow S_{M}^{(k)}(N) \quad \text { as } \quad T \rightarrow \infty .
$$

The rate of convergence depends on $N, M, k$ and the $\gamma_{n}$ 's.

The proof of this will be given at the end of this section.

Now we prove that the right-hand side of (1.1) is absolutely convergent. By (1.2) and (C.3), $|A(n, l)| \ll A(n, 2)$ for any $l \geq 2$ and by (C.1), $A(n, 1)=0$. Hence,

$$
\sum_{n_{1}, \cdots, n_{r}} \prod_{i=1}^{r}\left|A\left(n_{i}, l_{i}\right)\right| \ll \sum_{n_{1}, \cdots, n_{r}} \prod_{i=1}^{r} A\left(n_{i}, 2\right)=\left(\sum_{n} A(n, 2)\right)^{r}<\infty,
$$

by (C.2), and the right-hand side of (1.1) converges absolutely.

By (2.3) we get that for any $l \geq 1$,

(2.7) $\left|S_{M}(n, l)\right| \ll 1,|A(n, l)| \ll 1 \quad$ and $\quad S_{M}(n, l)-A(n, l) \ll_{l}\left\|a_{n}-\sigma_{n, M}\right\|$.

Using $\left|\prod_{i=1}^{r} x_{i}-\prod_{i=1}^{r} y_{i}\right| \ll_{r} \sum_{i=1}^{r}\left|x_{i}-y_{i}\right|$ when $\left|x_{i}\right|,\left|y_{i}\right| \ll 1$, we obtain from (2.6) and (2.7) that (recall that $c_{k}$ is the sum in the right side of (1.1))

$$
\begin{aligned}
& S_{M}^{(k)}(N)-c_{k} \\
\ll_{k} & \sum_{1 \leq r \leq k} \sum_{\substack{l_{1}, \ldots, l_{r} \geq 1 \\
l_{1}+\cdots+l_{r}=k}} \frac{k !}{l_{1} ! \cdots l_{r} !} \sum_{n_{1}<\cdots<n_{r} \leq N} \sum_{i=1}^{r}\left|S_{M}\left(n_{i}, l_{i}\right)-A\left(n_{i}, l_{i}\right)\right| \\
+ & \sum_{1 \leq r \leq k} \sum_{\substack{l_{1}, \cdots, l_{r} \geq 1 \\
l_{1}+\cdots+l_{r}=k}} \frac{k !}{l_{1} ! \cdots l_{r} !} \sum_{\substack{n_{1}, \cdots, n_{r}-1 \\
n_{r}>N}} \prod_{i=1}^{r}\left|A\left(n_{i}, l_{i}\right)\right| \\
\ll_{k} & N^{k-1} \sum_{n \leq N}\left\|a_{n}-\sigma_{n, M}\right\|+\sum_{n>N} A(n, 2) .
\end{aligned}
$$

For any $\epsilon>0$, in view of (C.2), we can fix an $N=N(\epsilon)>1$ such that

$$
\sum_{n>N} \int_{0}^{1} a_{n}(t)^{2} d t<\epsilon / 2
$$

According to (2.2) we take $M=M(N, \epsilon) \geq 1$ for which

$$
\left\|a_{n}-\sigma_{n, M}\right\|<\frac{\epsilon}{2} N^{-k} \quad \text { for all } n \leq N .
$$

For this value of $M$, both (2.8) and (2.4) are $\ll_{k} \epsilon$. By the triangle inequality, (2.8), (2.4) and Lemma 2, we conclude that

$$
m_{T}\left(\left(\sum_{n \leq N} a_{n}\left(\gamma_{n} t\right)\right)^{k}\right)-c_{k} \ll_{k} 3 \epsilon
$$

for all $T \geq T_{0}\left(\epsilon, \gamma_{1}, \cdots, \gamma_{N}\right)$. The proof of Lemma 1 is complete.

It remains to prove Lemma 2. From (2.5) and (2.1) it follows that

$$
S_{M}(n, l)=\sum_{\substack{\left|m_{1}\right|, \cdots,\left|m_{l}\right| \leq M \\ m_{1}+\cdots,+m_{l}=0}} c_{n, m_{1}} \cdots c_{n, m_{l}} .
$$


By (2.1),

$$
\begin{aligned}
& m_{T}\left(\left(\sum_{n \leq N} \sigma_{n, M}\left(\gamma_{n} t\right)\right)^{k}\right) \\
= & \sum_{n_{1}, \cdots, n_{k} \leq N} \sum_{\left|m_{1}\right|, \cdots,\left|m_{k}\right| \leq M} c_{n_{1}, m_{1}} \cdots c_{n_{k}, m_{k}} m_{T}\left(\prod_{i=1}^{k} e\left(m_{i} \gamma_{n_{i}} t\right)\right) .
\end{aligned}
$$

Now $m_{T}\left(\prod_{i=1}^{k} e\left(m_{i} \gamma_{n_{i}} t\right)\right)$ tends to 0 unless the linear combination $\sum_{i=1}^{k} m_{i} \gamma_{n_{i}}=0$, in which case the limit is 1 . Hence $m_{T}\left(\left(\sum_{n \leq N} \sigma_{n, M}\left(\gamma_{n} t\right)\right)^{k}\right)$ converges as $T \rightarrow \infty$. Indeed, as $N$ and $M$ are fixed integers, for any $\epsilon>0$,

$$
\left|m_{T}\left(\left(\sum_{n \leq N} \sigma_{n, M}\left(\gamma_{n} t\right)\right)^{k}\right)-L\right|<\epsilon
$$

for all $T \geq T_{0}\left(\epsilon, N, M, \gamma_{1}, \cdots, \gamma_{N}\right)$, where

$$
L=\sum_{n_{1}, \cdots, n_{k} \leq N} \sum^{*} c_{n_{1}, m_{1}} \cdots c_{n_{k}, m_{k}}
$$

and the summation $\sum^{*}$ runs over $\left|m_{1}\right|, \cdots,\left|m_{k}\right| \leq M$ for which $\sum_{i=1}^{k} m_{i} \gamma_{n_{i}}=0$. The proof of Lemma 2 will be complete after checking that $L=S_{M}^{(k)}(N)$.

Using the linear independence over $\mathbb{Q}$ of the $\gamma_{n}$ 's, when $\sum_{i=1}^{k} m_{i} \gamma_{n_{i}}=0$, the $\gamma_{n}$ 's can be partitioned into $r$ subsums (for some $r \leq k$ ) in which each subsum is of the form $\gamma_{n_{j}} \sum m_{v}$ with $\sum m_{v}=0$, and the $\gamma_{n_{j}}$ 's of different subsums are distinct. It follows that $L$ in (2.10) is equal to $S_{M}^{(k)}(N)$, by (2.9) and (2.6).

\section{Proof of Theorem 1}

We follow the argument in 3 to show that for any $\epsilon>0$,

$$
\left|\int_{-\infty}^{\infty} \alpha^{k} f(\alpha) d \alpha-T^{-1} \int_{0}^{T}\left(\sum_{n \leq N} a_{n}\left(\gamma_{n} t\right)\right)^{k} d t\right|<\epsilon
$$

for $N \geq N(\epsilon)$ and $T \geq T\left(\epsilon, N, \gamma_{1}, \cdots, \gamma_{N}\right)$. The proof is then completed by applying Lemma 1 .

For simplicity, denote

$$
S_{N}(t)=\sum_{n \leq N} a_{n}\left(\gamma_{n} t\right) \quad \text { and } \quad \mathcal{L}(\alpha, N)=\prod_{n \leq N} \int_{0}^{1} e\left(\alpha a_{n}(t)\right) d t .
$$

Following from [3, (3.1) and (3.5)] with $S_{N}(t)$ in place of $F(t)$, we have

$$
\left|m_{T}\left(p\left(S_{N}(t)\right)\right)-\int_{-A}^{A} \hat{p}(\alpha) \mathcal{L}(\alpha, N) d \alpha\right|<3 \epsilon
$$

for $A \geq A(\epsilon, \hat{p}), N \geq N(\epsilon, A)$ and $T \geq T(\epsilon, A, N)$. Here $p(\cdot)$ is any twice continuously differentiable function such that both $p$ and its Fourier transform $\hat{p}$ are absolutely integrable. From the proof of [3. Theorem 4] (see [3, p. 400]), replacing by a greater $A(\epsilon)$ and $N(\epsilon, A)$ if necessary, we obtain

$$
\left|\int_{-A}^{A} \hat{p}(\alpha) \mathcal{L}(\alpha, N) d \alpha-\int_{-\infty}^{\infty} p(\alpha) f(\alpha) d \alpha\right|<\epsilon
$$


for $A \geq A(\epsilon, \hat{p}), N \geq N(\epsilon, A)$. Choose now a twice continuously differentiable $p$ of compact support such that $p(\alpha)=\alpha^{k}$ for $|\alpha| \leq B$, and $|p(\alpha)| \leq|\alpha|^{k}$ for $|\alpha|>B$, as in the proof of [3, Theorem 6] (see [3, p. 397]). Then, for $B>2 c_{k+1} \epsilon^{-1}, N \geq$ $N_{0}(\epsilon), T \geq T_{0}(\epsilon, N)$, we have

$$
\left|m_{T}\left(p\left(S_{N}(t)\right)\right)-m_{T}\left(S_{N}(t)^{k}\right)\right| \leq B^{-1} m_{T}\left(S_{N}(t)^{k+1}\right) \leq 2 c_{k+1} B^{-1}<\epsilon,
$$

by Lemma 1, The fast decay of $f(\alpha)$ (see Theorem A) yields

$$
\left|\int_{-\infty}^{\infty} p(\alpha) f(\alpha) d \alpha-\int_{-\infty}^{\infty} \alpha^{k} f(\alpha) d \alpha\right|<\epsilon
$$

This proves (3.1), by adding the last two inequalities with (3.3) and (3.2).

\section{REFERENCES}

1. P.M. Bleher, Distribution of the error term in the Weyl asymptotics for the Laplace operator on a two-dimensional torus and related lattice problems, Duke Math. J. 70 (1993), 655-682. MR 1224102 (94g:11082)

2. P.M. Bleher, Z. Cheng, F.J. Dyson and J.L. Lebowitz, Distribution of the error term for the number of lattice points inside a shifted circle, Commun. Math. Phys. 154 (1991), 433-469. MR1224087 (94g:11081)

3. D.R. Heath-Brown, The distribution and moments of the error term in the Dirichlet divisor problem, Acta Arith. 60 (1992), 389-415. MR1159354 (93e:11114)

4. Y.-K. Lau, On the existence of limiting distributions of some number-theoretic error terms, J. Number Theory 94 (2002), 359-374. MR1916279 (2003e:11104)

5. K.-M. Tsang, Higher-power moments of $\Delta(x), E(t)$ and $P(x)$, Proc. London Math. Soc. (3) 65 (1992), 65-84. MR.1162488 (93c:11082)

6. W. Zhai, On higher-power moments of $\Delta(x)$ II, Acta Arith. 114 (2004), 35-54. MR2067871

Department of Mathematics, The University of Hong Kong, Pokfulam Road, Hong KONG

E-mail address: yklau@maths.hku.hk

Department of Mathematics, The University of Hong Kong, Pokfulam Road, Hong KONG

E-mail address: kmtsang@maths.hku.hk 